\title{
Random-Process Formulation of Computationally Efficient Performance Measures for Wideband Arrays in the Far Field*
}

\author{
Jeffrey O. Coleman \\ Naval Research Laboratory \\ Radar Division \\ Washington, DC \\ jeffc@alum.mit.edu
}

\author{
Robert J. Vanderbei \\ Princeton University \\ Dept. of Operations Research \\ and Financial Engineering \\ rvdb@princeton.edu
}

\begin{abstract}
Random-process autocorrelations and crosscorrelations in three dimensions here provide computationally efficient mean-square-error and average-gain measures to control main beam and sidelobe characteristics in convex optimization of far-field beamforming coefficients for large wideband arrays. An example optimized with the ultra-efficient interior-point code LOQO illustrates.
\end{abstract}

\section{Introduction}

Optimization of beamforming weights for wideband arrays of hundreds or thousands of sensor elements presents a formidable computational challenge, the far-field version of which we propose to address through formulation of a convex optimization problem-in particular a second-order cone program [1] - followed by its numerical solution with an ultra-efficient nonlinear optimizer, LOQO $[2,3]$, that is based on a so-called infeasible interior-point method. This paper develops linear and quadratic performance measures that can be used in efficient formulation of design constraints in such an optimization setting and illustrates the concepts through a simple LOQO-optimized example.

The unique contribution here is the approach, an extension to three dimensions of an approach developed earlier for the design of FIR digital filters in one dimension [4]. In this new setting we might control sidelobe levels, for example, by constraining the beamformer output power that would result from incident thermal radiation randomly distributed over wavenumbers corresponding to the desired

${ }^{*}$ This work was supported by the Office of Naval Research (through both institutions above), by National Science Foundation (NSF) grant MIP-9896034 to the University of Maryland Baltimore County (where Coleman is an Adjunct Assistant Professor of CS and Electrical Engineering), and by NSF grant DMS-9870317 to Princeton University sidelobe region. We will show that this is mathematically equivalent to constraining the RMS level of the array pattern over that sidelobe region. Similarly, fixing the crosscorrelation between the output of the beamformer and the output of a co-located isotropic element when both are subjected to thermal radiation randomly distributed over a desired mainbeam region effectively fixes the average main-beam gain.

The motivating advantage of this approach is computational efficiency. Beamformer optimization with secondorder cone programming has in fact been reported previously [5], but those early explorations used separate constraints at many individual points in wavenumber space. While such wavenumber sampling appears practically unavoidable in $L_{\infty}$ optimization, our experiments suggest that for $L_{2}$ optimization the present approach, by avoiding the implicit multidimensional numerical integration associated with sampling, can be two orders of magnitude faster for planar arrays of a few hundred elements.

The present approach of specifying thermal-radiation power density as a function of wavenumber also provides tremendous flexibility in the creation of systems of constraints. Here we only begin to tap this potential, illustrating the approach with wavenumbers uniformly distributed over the portion of a hemispherical surface corresponding to the desired sidelobe region for a narrowband array.

The key mathematical results are derived in the next section and illustrated through LOQO-based computational example in the third section.

\section{Beam Formulation}

Before dealing with thermal radiation, a single plane wave will help set up the concept of an array pattern in the precise way desired. 
The Array Pattern A single-frequency plane wave propagating at some velocity $c$ with wavenumber vector $-\ell$, the negative of a look vector $\ell$, has a complex field proportional to (we will say equal to) $e^{j 2 \pi(\ell \cdot \mathbf{x}+\|\ell\| c t)}$ at an arbitrary spatial location $\mathbf{x}$. (This field has spatial frequency $\|\ell\|$ and wavelength $1 /\|\ell\|$.) Multiplying this by some unitsconversion constant $\gamma$ results in the analytic signal at the output of an isotropic sensor at location $\mathbf{x}$, and more generally such a constant would take form $\gamma H(\ell)$ to reflect the additional gain and phase shift of a nonisotropic sensor. Complex sensor gain $H(\ell)$ is a function both of look direction $\ell /\|\ell\|$ and (temporal) frequency $c\|\ell\|$.

For the purposes of this paper, the beamformer output is a beamforming sum of the form ${ }^{1}$

$$
s_{b}(t)=\sum_{\mathbf{x} \in \mathcal{X}} w_{\mathbf{x}} H_{\mathbf{x}}(\ell) \gamma e^{j 2 \pi(\ell \cdot \mathbf{x}+\|\ell\| c t)}
$$

This analytic signal is formed as a complex linear combination of the analytic signals output from sensors, the array elements, at the locations in some finite set $\mathcal{X}$. This beam can be factored as $s_{b}(t)=B(\ell) \gamma e^{j 2 \pi\|\ell\| c t}$, where the array pattern or complex beamformer gain is

$$
B(\ell)=\sum_{\mathbf{x} \in \mathcal{X}} w_{\mathbf{x}} H_{\mathbf{x}}(\ell) e^{j 2 \pi \ell \cdot \mathbf{x}}
$$

This complex gain is the ratio of the analytic beamformeroutput signal to the analytic output of a hypothetical isotropic element at location $\mathbf{x}=0$.

A Random Field Now instead of a single plane wave, suppose the field comprises many plane-wave components, with the component at wavenumber $-\ell$ having random complex amplitude $d \mathcal{Z}(\ell)$, where random measure $\mathcal{Z}$ is finite in mean square (on any Borel set in $\mathbb{R}^{3}$ ) with orthogonal increments. Then the random incident field at location $\mathbf{x}$ and time $t$ is given by second-order stochastic integral ${ }^{2}$

$$
\int e^{j 2 \pi(\ell \cdot \mathbf{x}+\|\ell\| c t)} d \mathcal{Z}(\ell)
$$

and yields beamformer output

$$
s_{b}(t)=\gamma \int B(\ell) e^{j 2 \pi(\|\ell\| c t+\ell \cdot \mathbf{x})} d \mathcal{Z}(\ell)
$$

As an example, suppose for some particular fixed $\ell_{0}$ that $\mathcal{Z}(B)$ is defined to yield a zero-mean circular complex random variable if $\ell_{0} \in B$ and so that $\mathcal{Z}(B)=0$

\footnotetext{
${ }^{1}$ More generally, the weights can be made functions of frequency.

${ }^{2}$ Many texts like Wong and Hajek [6], Rosenblatt [7], Adler [8], and Yaglom [9] use a Stieltjes-style integral with respect to an orthogonalincrement process, but that is more natural in a single dimension. The present development instead follows Rozanov [10] and uses a Lebesguestyle integral with respect to a random measure.
}

if $\ell_{0} \notin B$. In this simple case, the field then comprises a single plane wave at wavenumber $-\ell_{0}$, but with random (Rayleigh-distributed) amplitude and (uniformly distributed) phase. With a more-general random measure $\mathcal{Z}$, the random field (2) would comprise uncorrelated components distributed across the spectrum of wavenumbers.

The Power Gain The average power $P_{b}$ of this beamformer output is

$$
\begin{aligned}
P_{b} & =E\left\{s_{b}(t) \overline{s_{b}(t)}\right\} \\
& =|\gamma|^{2} \iint B(\ell) \overline{B(\mu)} e^{j 2 \pi[(\|\ell\|-\|\mu\|) c t+(\ell-\mu) \cdot \mathbf{x}]} \\
& \times E\{d \mathcal{Z}(\ell) \overline{d \mathcal{Z}(\mu)}\}
\end{aligned}
$$

using basic result $E\{d \mathcal{Z}(\ell) \overline{d \mathcal{Z}(\mu)}\}=\delta(\ell-\mu) d \mu d F(\ell)$ from stochastic integration theory $[6,9]$. Since the intensity $E\left\{|\cdot|^{2}\right\}$ of the random field given by (2) is similarly given by $\int d F(\ell)=F\left(\mathbb{R}^{3}\right)$, positive Borel measure $F$ on $\mathbb{R}^{3}$ should be interpreted as the distribution versus look vector of complex field intensity.

A normalization further clarifies the picture. The normalization constant is the power $P_{i}$ out of an isotropic element at $\mathbf{x}=0$, obtained by replacing $B(\ell)$ with unity in the derivation of (3) to obtain

$$
P_{i}=E\left\{s_{i}(t) \overline{s_{i}(t)}\right\}=|\gamma|^{2} F\left(\mathbb{R}^{3}\right)
$$

The power gain of the beamformer in response to the hypothesized random incident field is the ratio

$$
\frac{P_{b}}{P_{i}}=\int|B(\ell)|^{2} d \mathcal{P}(\ell)
$$

where normalized measure $\mathcal{P}$, specified by $\mathcal{P}(B)=$ $F(B) / F\left(\mathbb{R}^{3}\right)$, is seen to be a probability measure. This power gain can therefore be effectively interpreted as a weighted mean-square beamformer gain. Many useful performance measures can be formulated through wavenumber distributions specified by $\mathcal{P}$ or $F$.

Normalized Crosscorrelation If we adopt the awkward but consistent notation $P_{b i}$ for the crosscorrelation between the beamformer and isotropic outputs, then a completely parallel derivation yields

$$
P_{b i}=E\left\{s_{b}(t) \overline{s_{i}(t)}\right\}=|\gamma|^{2} \int B(\ell) d F(\ell)
$$

and

$$
\frac{P_{b i}}{P_{i}}=\int B(\ell) d \mathcal{P}(\ell)
$$

a weighted-average beamformer gain. This can be useful in main-beam specifications. 


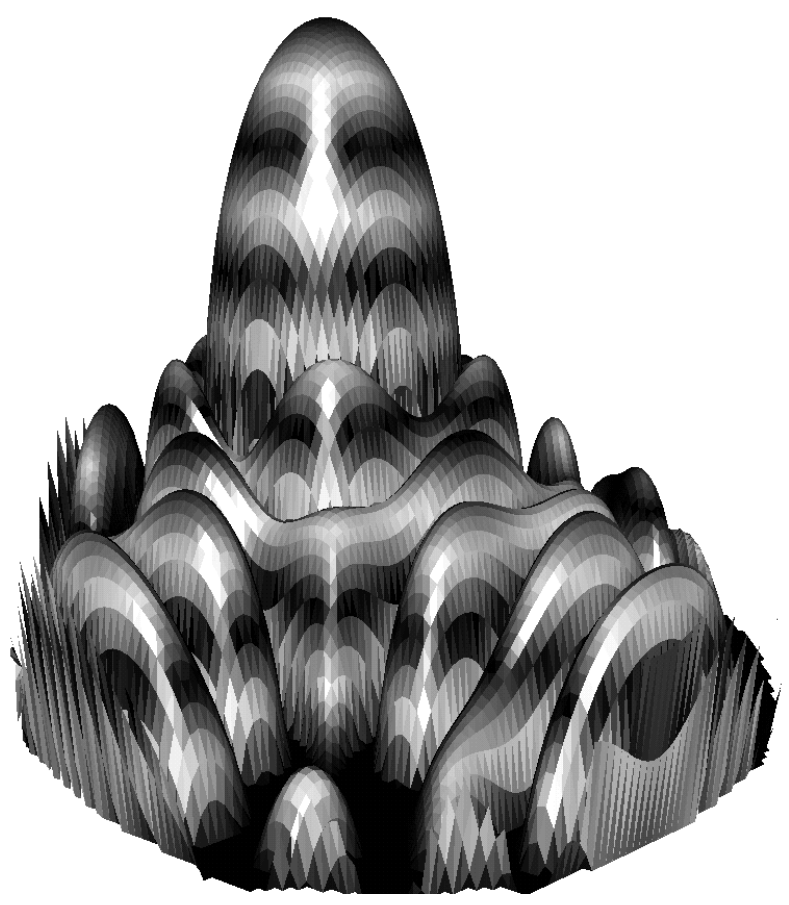

Figure 1. Simple example: A circular off-boresight main beam and minimum-RMS sidelobes.

The Autocorrelation of the Field It is sometimes advantageous to compute average power $P_{b}$ and crosscorrelation $P_{b i}$ in a "spatial domain" obtained through inverse Fourier transformation of the relationships (3-5). Begin by substituting (1) into (3) to obtain

$$
\begin{aligned}
P_{b} & =|\gamma|^{2} \sum_{\mathbf{x}, \mathbf{y} \in \mathcal{X}} w_{\mathbf{x}} \overline{w_{\mathbf{y}}} \int H_{\mathbf{x}}(\ell) \overline{H_{\mathbf{y}}(\ell)} e^{j 2 \pi \ell \cdot(\mathbf{x}-\mathbf{y})} d F(\ell) \\
& =|\gamma|^{2} \sum_{\mathbf{x}, \mathbf{y} \in \mathcal{X}} w_{\mathbf{x}} \overline{w_{\mathbf{y}}}\left(h_{\mathbf{x}} \star R \star \widetilde{h}_{\mathbf{y}}\right)(\mathbf{x}-\mathbf{y})
\end{aligned}
$$

where $\star$ denotes convolution, where $h_{\mathbf{y}}(\mathbf{x})$ is the inverse Fourier transform of $H_{\mathbf{y}}(\ell)$, where $\widetilde{h}_{\mathbf{y}}$ denotes the paraconjugate of function $h_{\mathbf{y}}$, related according to $\widetilde{h}_{\mathbf{x}}(\mathbf{z})=$ $\overline{h_{\mathbf{x}}(-\mathbf{z})}$, and where $R(\mathbf{z})$ is the $t=0$ spatial autocorrelation of the random field:

$$
\begin{aligned}
R(\mathbf{z}) & =E\left\{\int e^{j 2 \pi \ell \cdot \mathbf{y}} d \mathcal{Z}(\ell) \overline{\int e^{j 2 \pi \mu \cdot(\mathbf{y}-\mathbf{z})} d \mathcal{Z}(\mu)}\right\} \\
& =\int e^{j 2 \pi \ell \cdot \mathbf{z}} d F(\ell)
\end{aligned}
$$

Unsurprisingly, this is just the inverse Fourier transform of the distribution $F$ of power across the look-vector spectrum. Parallel derivations yield $P_{i}=|\gamma|^{2} R(0)$ and

$$
P_{b i}=|\gamma|^{2} \sum_{\mathbf{x} \in \mathcal{X}} w_{\mathbf{x}}\left(h_{\mathbf{x}} \star R\right)(\mathbf{x})
$$

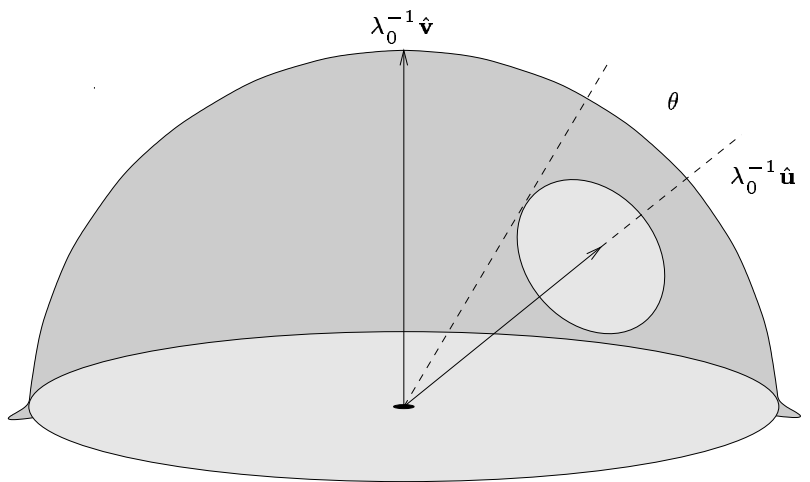

Figure 2. For narrowband sidelobe control, look vector $\ell$ was distributed uniformly on a hemisphere with a main-beam cutout.

The importance of these forms in computing the power ratios lies in the finite character of the sums and in special cases permitting the convolutions to be easily and exactly computed. The most obvious is the isotropic array-element case $h_{\mathbf{x}}(\mathbf{z})=\delta(\mathbf{z})$ in which the convolutions disappear and leave only the autocorrelation. This is the case developed in the example.

\section{A Simple Computational Example}

We consider a narrowband array operating at temporal frequency $c / \lambda$ with 61 array elements located in the horizontal plane (orthogonal to $\hat{\mathbf{v}}$ ) within an origin-centered, hexagonally shaped region at the $\lambda / 2$ spaced points of a triangular lattice. The LOQO-optimized array pattern in Fig. 1 minimizes sidelobe power gain $P_{b} / P_{i}$ using (6) while fixing the average main-beam gain $P_{b i} / P_{i}$ using (7).

Sidelobe power gains are computed supposing that the random-field look vectors are distributed as sketched in Fig. 2, uniformly on the upper surface of a radius $\lambda^{-1}$ hemisphere less a circular cutout of half angular width $\theta=20^{\circ}$ and centered in the direction $\hat{\mathbf{u}}$ of the desired main beam. Here $\hat{\mathbf{u}}$ and $\hat{\mathbf{v}}$ are $30^{\circ}$ apart, so the main beam is $30^{\circ}$ "off boresight." The uniform spectral surface density chosen integrates to unit power. The average main-beam gain is computed supposing the random-field look vectors are distributed instead over the cutout region in the figure, again with power normalization.

The autocorrelation kernel $R$ in (7) is just the inverse Fourier transform of a uniform distribution on this cutout, and the kernel $R$ in (6) is the difference between the inverse Fourier transforms of uniform distributions on the hemisphere and on the cutout. Each transform is computed using the result derived in the appendix. For example, with $\underline{\mathbf{x}}$ a dimensionless location normalized by $\lambda$ for mathematical 
convenience, the autocorrelation is given by

$$
\begin{aligned}
2 R(\lambda \underline{\mathbf{x}}) & =\int_{0}^{1} J_{0}\left(2 \pi \sqrt{1-\phi^{2}}\|\underline{\mathbf{x}}-\hat{\mathbf{v}} \cdot \underline{\mathbf{x}}\|\right) e^{i 2 \pi \phi \hat{\mathbf{v}} \cdot \underline{\mathbf{x}}} d \phi \\
& -\int_{\cos \theta}^{1} J_{0}\left(2 \pi \sqrt{1-\phi^{2}}\|\underline{\mathbf{x}}-\hat{\mathbf{u}} \cdot \underline{\mathbf{x}}\|\right) e^{i 2 \pi \phi \hat{\mathbf{u}} \cdot \underline{\mathbf{x}}} d \phi
\end{aligned}
$$

For each $\ell$, the decibel gain $20 \log |B(\ell)|$ in Fig. 1 is plotted versus the projection of $\ell$ into the horizontal plane. Since the look vectors of interest are all of length $\lambda^{-1}$, those projected points form a filled circle, the base of the plot. The image gray scale cycles from black to white and back to black every $10 \mathrm{~dB}$, so the sidelobes closest to the main beam peak about $-25 \mathrm{~dB}$, whereas the largest of the sidelobes close to the front (to the viewpoint of the plot) are at about $-35 \mathrm{~dB}$.

\section{APPENDIX: Inverting a Spherical-Shell Spectrum}

Here we will multiply look vectors by $\lambda$ and locations by $\lambda^{-1}$ and in either case denote the dimensionless normalized result with an underscore. We will denote dimensionless unit vectors with a hat.

Suppose we are given some unit vector $\hat{\mathbf{v}}$ and some function $S(z)$ that together define spectral measure $F$ through $d F(\ell)=(4 \pi)^{-1} S(\underline{\ell} \cdot \hat{\mathbf{v}}) d A(\underline{\ell})$, using normalized look vector $\underline{\ell}=\ell \lambda$ and with $A$ representing Lebesgue surface-area measure on hollow unit sphere $\mathcal{S}$ in $\mathbb{R}^{3}$ and extended to $\mathbb{R}^{3}$ with $A\left(\mathcal{S}^{c}\right)=0$. The autocorrelation is then

$$
R(\mathbf{x})=\int e^{i 2 \pi \ell \cdot \mathbf{x}} d F(\ell)=\frac{1}{4 \pi} \int S(\underline{\ell} \cdot \hat{\mathbf{v}}) e^{i 2 \pi \underline{\ell} \cdot \underline{\mathbf{x}}} d A(\underline{\ell}),
$$

where $\underline{\mathbf{x}}=\lambda^{-1} \mathbf{x}$. Note that when $S(z)=1$ everywhere, the normalization has resulted in $R(0)=A(\mathcal{S})=1$.

To put this integral in a more convenient form for computation, begin by expressing vectors $\underline{\ell}$ and $\underline{\mathrm{x}}$ in a for$\mathrm{m}$ of cylindrical coordinates with axis $\hat{\mathbf{v}}$ by decomposing each into a component along $\hat{\mathbf{v}}$ and a component in the two-dimensional subspace orthogonal to $\hat{\mathbf{v}}$. This results in $\underline{\ell}=\phi \hat{\mathbf{v}}+\ell_{\perp}$ and $\underline{\mathbf{x}}=\xi \hat{\mathbf{v}}+\mathbf{x}_{\perp}$. Then write $\ell_{\perp}$ as $\rho \hat{\mathbf{h}}$ with $\rho>0$ so that $\underline{\ell}=\phi \hat{\mathbf{v}}+\rho \hat{\mathbf{h}}$. (The $\rho=0$ point can be left undefined with no effect on the integral.) These decompositions transform the integrand to $S(\phi) e^{i 2 \pi \phi \xi} e^{i 2 \pi \rho \hat{\mathbf{h}} \cdot \mathbf{x}_{\perp}}$.

Determining the normalized differential surface area $d A(\underline{\ell})$ is more involved. Constraint $\underline{\ell} \in \mathcal{S}$ implies both $d \underline{\ell} \perp \underline{\ell}$ and $d \hat{\mathbf{h}} \perp \hat{\mathbf{h}}$. The first of these, using $d \underline{\ell}=$ $d \phi \hat{\mathbf{v}}+\rho d \hat{\mathbf{h}}+d \rho \hat{\mathbf{h}}$, becomes $\underline{\ell} \cdot d \underline{\ell}=\phi d \phi+\rho d \rho=0$ with help from the second. This implies $d \rho=-(\phi / \rho) d \phi$ and hence $d \underline{\ell}=\rho d \hat{\mathbf{h}}+(\hat{\mathbf{v}}-(\phi / \rho) \hat{\mathbf{h}}) d \phi$. Now $d \hat{\mathbf{h}}$ and $d \phi$ can be seen to contribute to changes in $d \underline{\ell}$ in orthogonal directions, so $d A(\underline{\ell})=\rho d \hat{\mathbf{h}} \times\|(\hat{\mathbf{v}}-(\phi / \rho) \hat{\mathbf{h}})\| d \phi$. But $\|(\hat{\mathbf{v}}-(\phi / \rho) \hat{\mathbf{h}})\|^{2}=1+(\phi / \rho)^{2}=\|\underline{\ell}\|^{2} / \rho^{2}$ and hence $d A(\underline{\ell})=\|\underline{\ell}\| d \hat{\mathbf{h}} d \phi$. In fact $\|\underline{\ell}\|=1$ is all that remains to respect $\underline{\ell} \in \mathcal{S}$, so $d A(\underline{\ell})=d \hat{\mathbf{h}} d \phi$.

Putting these results together, the integral becomes

$$
R(\mathbf{x})=\frac{1}{2} \int_{-1}^{1} S(\phi) e^{i 2 \pi \phi \xi}\left[\frac{1}{2 \pi} \int_{\mathbb{T}} e^{i 2 \pi \rho \hat{\mathbf{h}} \cdot \mathbf{x}_{\perp}} d \hat{\mathbf{h}}\right] d \phi,
$$

where $\mathbb{T}$ denotes the unit circle in $\mathbb{R}^{2}$. The inner integral is a special case of standard [11] result $J_{n}(w)=$ $\frac{1}{2 \pi i^{n}} \int_{0}^{2 \pi} e^{i w \cos \theta} e^{i n \theta} d \theta$, so

$$
R(\mathbf{x})=\frac{1}{2} \int_{-1}^{1} S(\phi) J_{0}\left(2 \pi \rho(\phi)\left\|\mathbf{x}_{\perp}\right\|\right) e^{i 2 \pi \phi \xi} d \phi,
$$

where the dependence of $\rho$ on $\phi$ through the requirement that $\underline{\ell}=1$ has been shown explicitly. It looks slightly nicer written as

$$
R(\mathbf{x})=\frac{1}{2} \int_{-1}^{1} S(\phi) J_{0}\left(2 \pi\left\|\ell_{\perp}\right\|\left\|\mathbf{x}_{\perp}\right\|\right) e^{i 2 \pi \phi \xi} d \phi .
$$

\section{References}

[1] M. Lobo, L. Vandenberghe, S. Boyd, and H. Lebret, "Applications of second-order cone programming," Linear Algebra and its Applications, vol. 284, pp. 193-228, Nov. 1998.

[2] R. Vanderbei, "LOQO: An interior point code for quadradic programming," Technical Report SOR 94-15, Princeton University, 1994, revised 11/30/98, to appear in Optimization Methods and Software.

[3] R.J. Vanderbei and D.F. Shanno, "An interior-point algorithm for nonconvex nonlinear programming," Tech. Rep. SOR-97-21, Statistics and Operations Research, Princeton University, 1997, To appear in Computational Optimization and Applications.

[4] J. O. Coleman, "Systematic mapping of quadratic constraints on embedded FIR filters to linear matrix inequalities," in Proc. 1998 Conf. on Information Sciences and Systems (CISS '98), Princeton, NJ, Mar. 1998.

[5] H. Lebret and S. Boyd, "Antenna array pattern synthesis via convex optimization," IEEE Trans. Signal Processing, vol. 45, no. 3, pp. 526-532, Mar. 1997.

[6] Eugene Wong and Bruce Hajek, Stochastic Processes in Engineering Systems, Springer-Verlag, New York, 1985.

[7] Murray Rosenblatt, Stationary Sequences and Random Fields, Birkhäuser Boston, Boston, 1985.

[8] Robert J. Adler, The Geometry of Random Fields, Wiley, New York, 1981.

[9] A. $\widetilde{M}$. Yaglom, Correlation Theory of Stationary and Related Random Functions, Volume 1: Basic Results, SpringerVerlag, New York, 1987.

[10] Yu A. Rozanov, Stationary Random Processes, Holden-Day, San Francisco, 1967.

[11] Eric W. Weisstein, Concise CRC Encyclopedia of Mathematics, CRC Press, 1998. 